\title{
Improving Electronic Health Records Training through Usability Evaluation in Primary Care
}

\section{Min Soon Kim ${ }^{1,2 *}$}

${ }^{1}$ Department of Health Management and Informatics, School of Medicine, University of Missouri, USA

${ }^{2}$ University of Missouri Informatics Institute, University of Missouri, USA

\section{Introduction}

Federal leadership has been driving electronic health record (EHR) adoption across the US, with hopes of relieving financial burden in health care and providing better care. An EHR is a systematic, digital record system that collects patient health information. According to the recent statistics in 2012 by the Centers for Disease Control and Prevention, National Center for Health Statistics (CDC NCHS), over $70 \%$ of office-based physicians used an any form of EHR system in their practices to fulfill the Meaningful Use objectives [1]. Successful adoption of EHR systems has the potential to enhance quality of medical care by streamlining clinical workflow and by providing clinical functionality, for example, calculation of appropriate drug dosages for patients with complex cases. EHR applications, however, should not only fulfill functionality requirements, but also employ non functional requirement, such as user interfaces that are designed to improve the user experience, and consequently improve the clinical workflow process.

Recent research has constantly emphasized the challenges of EHR implementation across health care organizations. Conversely, many negative effects have been documented, which include: lower effectiveness, decreased efficiency, decreased team collaboration, increased cognitive load, medical errors, and decreased quality of patient care. These issues can be related to the scope of usability, "effectiveness, efficiency and satisfaction that users of a specific product can achieve, to a certain defined level, with an explicit set of tasks in a specific environment" [2]. While it is critical to address usability issues in a timely manner by changing their EHR features or functionalities, current improvement process takes substantial time and effort, especially in large health care setting; to evaluate the changes before they are implemented before the end user.

Primary care physicians (family medicine and general internal medicine) account for a majority of patient visits for preventive and ongoing healthcare, encompassing a range of conditions. They practice in a highly interruptive, time-pressured environment [3]. Rapid medical advances and socio-demographic change call greater primary care performance, which demands efficient use of information management through an EHR [4].

Many studies of successful EHR implementation have constantly emphasized the importance of early and timely training for successful implementation [5]. Residents are in-training medical professionals holding a medical degree (MD, DO) who will be full-time staff clinicians after training. Most residencies in primary care run a three-year program. In each year of training, the residents are given increasing levels of privilege and responsibility under supervision by senior residents and attending physicians. Residents are involved in performing diagnostic and treatment procedures appropriate to their medical specialty and level of training. Aaronson et al., based on survey of 219 family practice residency programs about the use of EHR systems in the residency program, emphasized that the training the residents received may influence not only the perceived ease of the EHR application, but attitudes concerning the capability of the EHR, such as time management and medical record accuracy in their practices after residency [6].

However, according to the "2012 EHR User Satisfaction Survey from 3,088 family physicians," nearly $62 \%$ of the respondents were not satisfied with many of the best-known EHR systems, with the area of lowest satisfaction in EHR vendor support and training [7]. While medical school and teaching hospitals have a responsibility to teach residents how to use EHRs in the context of providing sound clinical care, there is little guidance for effective strategies for training learners how to use EHRs for the patient care [8-10]. Moreover, current EHRs require an enormous investment of resources for the user to reach a certain level of proficiency (learning curve), which is a major barrier for beginning physicians (e.g., residents) who are not adequately trained in their medical schools for use of EHR. In an effort to relieve learning curve issues, many hospitals and clinics offer intensive EHR trainings for their residents. However, it is a challenge to find adequate time to train busy physicians in ambulatory care settings to use new EHR systems, target training to users' needs, and provide hands-on, on-site support.

In a hope to improve training program for their physicians, a study explored the relationship between usability evaluation and training of a commercial EHR system at an urban medical center in NYC [11]. A task-based usability test was conducted in a laboratory setting for approximately a month after a four hour in-class training session, involving 5 physician end users without prior EHR experience other than the system under study. Through usability evaluation analyses, the study identified areas of improvement where physician users encountered problems as they completed their tasks. The study not only found many areas of potential enhancement for system usability and learnability but also suggested recommendations for enhancing current EHR training. The study findings demonstrated that there would be a potential benefit of usability evaluation methodology in improving EHR training.

While use of EHRs is becoming an integral constituent of medical skills, EHRs with steep learning curves could make new residents in primary care, who are already overwhelmed in learning a new specialty,

${ }^{*}$ Corresponding author: Min Soon Kim, Assistant Professor, Department of Health Management and Informatics University of Missouri School of Medicine, Core Faculty, University of Missouri Informatics Institute CE728 Clinical Support \& Education, DC006.00, Five Hospital Drive, Columbia, MO 65212, USA, Tel: +1.573.884.0115; Fax +1.573.882.6158; E-mail: kimms@health.missouri.edu

Received August 22, 2013; Accepted August 23, 2013; Published August 28, 2013

Citation: Kim MS (2013) Improving Electronic Health Records Training through Usability Evaluation in Primary Care. J Health Med Informat 4: e110. doi:10.4172/2157-7420.1000e110

Copyright: () $2013 \mathrm{Kim}$ MS. This is an open-access article distributed under the terms of the Creative Commons Attribution License, which permits unrestricted use, distribution, and reproduction in any medium, provided the original author and source are credited. 
Citation: Kim MS (2013) Improving Electronic Health Records Training through Usability Evaluation in Primary Care. J Health Med Informat 4: e110. doi:10.4172/2157-7420.1000e110

Page 2 of 2

cope with poorly designed EHR use requirements. Thus, identifying and addressing early barriers in the learning environment through usability evaluation can help improve overall capacity of the new physicians, and save costs for the organization.

\section{References}

1. Hsiao CJ, Hing E (2012) Use and characteristics of electronic health record systems among office-based physician practices: United States. NCHS Data Brief 1-8.

2. Mary FT (2007) Common industry specification for usability requirements NISTIR 7432.

3. Schoen C, Osborn R, Huynh PT, Doty M, Peugh J, et al. (2006) On The Front Lines Of Care: Primary Care Doctors Office Systems, Experiences, and Views in Seven Countries. Health Aff 25: w555-571.

4. Grumbach K, Bodenheimer T (2002) A primary care home for Americans: putting the house in order. JAMA 288: 889-893.
5. Anderson LK, Stafford CJ (2002) The "big bang" implementation: not for the faint of heart. Comput nurs 20: 14-20.

6. Aaronson JW, Murphy-Cullen CL, Chop WM, Frey RD (2011) Electronic medical records: the family practice resident perspective. Fam Med 33: 128-132.

7. Edsall RL, Adler KG (2011) The 2011 EHR User Satisfaction Survey: responses from 2,719 family physicians. Fam Pract Manag 18: 23-30.

8. Peled JU, Sagher O, Morrow JB, Dobbie AE (2009) Do electronic health records help or hinder medical education? PLoS Med 6: e1000069.

9. (1998) Report II: Contemporary Issues in Medicine: Medical Informatics and Population Health. Medical School Objectives Project.

10. Gliatto P, Masters P, Karani R (2009) Medical student documentation in the medical record: is it a liability? Mt Sinai J Med 76: 357-364.

11. Kushniruk AW, Myers K, Borycki EM, Kannry J (2009) Exploring the relationship between training and usability: a study of the impact of usability testing on improving training and system deployment. Stud Health Technol Inform 143: 277-283. 